Results We obtained 346 records related to the DRGs selected: 101 (29\%) ADRs and Testing Oral Exposure to Drugs, 91 (27\%) poisoning, 20 (6\%) drug abuse, 7 (2\%) reactions to foods and $97(28 \%)$ unspecified events. It was possible to identify the drug involved in only 51 records: antibiotics, NSAIDs, chemotherapy agents, local anaesthetics, opioids and immunoglobulin were the agents mainly reported. Only 2 cases had been reported to the Pharmacovigilance Office and entered in the Italian National Pharmacovigilance Database.

Conclusions Our survey shows a mismatch between the ADRs documented in the hospital discharge records and those actually reported to the hospital's Pharmacovigilance Office, highlighting the problem of under-reporting. The data could be useful for implementing measures to raise awareness among health care professionals and to spread the culture of drug safety.

No conflict of interest.

\section{GRP-189 UPGRADING A VITAMIN K ANTAGONIST CONSULTATION PROGRAMME: IDENTIFICATION OF NEW ORAL ANTICOAGULANT (NOAC) PRESCRIPTION PARTICULARITIES}

doi:10.1136/ejhpharm-2013-000276.189

${ }^{1} \mathrm{AG}$ Caffin, ${ }^{2} \mathrm{~V}$ Algalarrondo, ${ }^{2} \mathrm{~S}$ Dinanian, ${ }^{1} \mathrm{~A}$ Rieutord, ${ }^{1} \mathrm{R}$ Haddad, ${ }^{1} \mathrm{C}$ Courtin. ${ }^{1}$ Hôpital Antoine Béclère, Pharmacy, Clamart, France; ${ }^{2 H o ̂ p i t a l ~ A n t o i n e ~ B e ́ c l e ̀ r e, ~ C a r d i o l o g y, ~}$ Clamart, France

Background Our pharmacy department performs 150 Vitamin K antagonist (VKA) patient consultations annually. New oral anticoagulants (NOACs) are expected to replace VKAs in most of their indications. The variety of drugs and the different therapeutic schemes depending on the indications can be extremely hazardous. The NOAC marketing authorization (MA) came along with a European risk management plan.

Purpose To assess the prescription particularities of NOACs, further to the extension of their indication in cardiology in the management of atrial fibrillation (European Society Of Cardiology Guidelines in 2012).

Materials and Methods A retrospective study of NOAC prescriptions was performed from January 2011 till July 2012 to identify the main departments prescribing them and to evaluate the indications. Secondly, we questioned 2 cardiologists to determine the needs of patients and other healthcare practitioners for information about these treatments.

Results An increase in NOAC prescriptions was observed: 25 in 2011 and 41 in 2012 (7 months). The main prescribing departments were cardiology and orthopaedic surgery with respectively 48 and 12 patients. 18 prescriptions (2011) vs. 8 in 2012 did not match the recommendations. This was mainly due to prescription anticipating the MA in cardiology. Information needs identified by the cardiologists concerned prescription (switching from VKA-NOAC, effects of medicines altering the haemostasis and changing the dose required, perioperative management for optimal safety if the patient needs surgery or invasive procedures). The patient also needs to be informed (knowledge of the treatment, awareness of the risk of haemorrhage, self-medication and clinical surveillance of any bleeding).

Conclusions This preliminary research shows that it is necessary to supervise NOAC prescriptions and inform patients, to ensure these new treatments will be used properly. It allowed us to design a standard protocol for prescribing and monitoring NOAC. Our anticoagulant consultation programme will include these needs and NOAC patient consultation will be offered from January 2013.

No conflict of interest.

\section{GRP-190 USE OF TRANQUILLISERS AND RESTRAINT IN A FRENCH TEACHING ACUTE CARE HOSPITAL}

doi:10.1136/ejhpharm-2013-000276.190

${ }^{1} \underline{N}$ Curatolo, ${ }^{2 B}$ Colombier, ${ }^{3 T}$ Chinet, ${ }^{4} 0$ Goeau-Brissonniere, ${ }^{2} \mathrm{~L}$ Teillet, ${ }^{1} \mathrm{~F}$ Lemercier, ${ }^{2} \mathrm{~S}$ Moulias. 'Hôpital ambrsoie Paré, Pharmacy, Boulogne Billancourt, France; ${ }^{2}$ Hôpital ambrsoie Paré, Geriatrics, Boulogne Billancourt, France; '3hôpital ambrsoie Paré, Pneumology, Boulogne Billancourt, France; ${ }^{4}$ Hôpital ambrsoie Paré, Vascular surgery, Boulogne Billancourt, France

Background Major and minor tranquillisers can be used to chemically restrain a patient. Use of chemical restraint (CR) has been described mainly in long care settings but there is very limited information when considering acute care hospitals.

Purpose To study the prescriptions for major and minor tranquillisers in 3 clinical wards of a French teaching hospital and to determine if they can be considered CR.

Materials and Methods This prospective study took place over 2 weeks in 3 different wards: geriatrics, pneumology and vascular surgery. Tranquilizers were defined as anxiolytics (minor) and neuroleptics (major). Prescriptions were checked daily and for each patient with a tranquilliser, medical records were screened to determine whether it was newly prescribed. For every newly-prescribed tranquilliser the practitioner was asked the indication, if he considered his prescription was a CR and if the patient was being physically restrained.

Results $45.2 \%$ of the 137 patients included had been prescribed at least 1 tranquilliser. $54.5 \%$ of the 77 tranquillisers prescribed were introduced during the hospitalisation. Among those 42 newlyintroduced tranquillisers, $9(21.4 \%)$ were considered as CR by the prescribers. $6.6 \%$ of the patients included were chemically restrained, which is comparable with previous retrospective studies of restraint in acute care wards. The most frequently prescribed CR was alprazolam $(55.6 \%)$ and the most frequent indication for CR was anxiety. In addition $88.9 \%$ of the CR drugs were prescribed "when required' leaving responsibility for administration to nurses alone. None of the patients with tranquillisers had physical restraint.

Conclusions This is the first prospective study on restraint in an acute care hospital. CR is used for a minority of patients; however it is mostly prescribed 'when required'. Hence it should be used with the utmost care and prescribed with the most precise instructions in order to avoid misuse and risk of abuse.

No conflict of interest.

\section{GRP-191 WOULD 50ML PREFILLED SYRINGES IMPROVE PATIENT SAFETY? OBSERVATION OF 960 INFUSIONS WITH A SYRINGE PUMP IN A MULTI-CENTRIC STUDY}

doi:10.1136/ejhpharm-2013-000276.191

'F Schwarzenbach, 'C Berteau, ${ }^{2} \mathrm{~F}$ Urvoy, ${ }^{1} \mathrm{PE}$ Laurent. ${ }^{1 B D}$, Medical Affairs, Le Pont de Claix, France; ${ }^{2} B D$, Strategic Marketing, Le Pont de Claix, France

Background Before infusion with a syringe pump, drug preparation requires often dilution and more steps compared to most other injection practises, thus involves risks for patients and Health Care Workers (HCWs). The literature indicates that prefilled syringes (PFSs) address these issues successfully but most data do not apply to intravenous infusions.

Purpose BD ran a multicentre study to evaluate the expected impacts of a new BD Sterifill $50 \mathrm{ml}$ PFS on patient and HCW safety, comparing an infusion with a syringe pump using either the PFS or a conventional system (drug in ampoule, diluent, $50 \mathrm{ml}$ syringe filled at time of use).

Materials and Methods $120 \mathrm{HCWs}$ performed infusions in a randomised order, 4 with the new PFS, 4 with the conventional system, mimicking regular dobutamine preparation and infusion $(250 \mathrm{mg} /$ $50 \mathrm{ml}, 10 \mathrm{ml} / \mathrm{h}$ ). For all 960 cases, an observer recorded any handling 
errors. Results were analysed by sub-groups using FDA Human Factors guidelines. A risk score was calculated for each syringe type and for each step based on the error occurrence and its severity according to the risk class: dosing error, microbial contamination and unexpected adverse event. This Health Hazard Risk Evaluation (HHRE) method has been published by BD.

Results With PFSs the handling error rate was lower and the HHRE score was better. Dosing error and microbial contamination occurred respectively in $12.7 \%$ and $43.1 \%$ of infusions with the conventional system but only in $4.8 \%$ and $0.2 \%$ with PFSs. $6 \%$ of conventional system infusions showed a risk of needle stick injury (one injury actually happened) versus $0 \%$ for PFS.

$84 \%$ of HCWs would use the new PFS in their daily practise mainly to decrease the risk of contamination and administration errors, and to save time.

Conclusions Prefilled $50 \mathrm{ml}$ infusions with a syringe pump help reduce patient risks (especially dose error and contamination) and $\mathrm{HCW}$ injuries. PFS is also preferred by HCWs.

No conflict of interest.

\section{Technology (including: robots for production, incompatibilities, drug production and analytics, CRS)}

\section{TCH-001 A CASE REPORT OF A WOMAN HOSPITALISED FOR SEVERE LOSS OF WEIGHT AND PSYCHOTIC DECOMPENSATION AFTER TAKING A SLIMMING PREPARATION}

doi:10.1136/ejhpharm-2013-000276.192

${ }^{1} \mathrm{~N}$ Layoun, ${ }^{1} \mathrm{C}$ Blanco, 'A Benassaya, ${ }^{2} \mathrm{H}$ Rebiere, ${ }^{2} \mathrm{C}$ Civade. ${ }^{1} \mathrm{CHRU}$ Montpellier, Pharmacy, Montpellier, France; ${ }^{2}$ French Health Agency, Laboratories and Control Directorate, Vendargues, France

Background A French pharmacovigilance centre recorded the case of a 40-year-old woman hospitalised for severe loss of weight $(16 \mathrm{~kg}$ in 3 months) associated with hypokalaemia, inflammatory syndrome and psychotic decompensation, after taking a slimming preparation. It was sold on the internet as an herbal medicine containing natural authorised substances.

Purpose The expertise of the French Health Agency (ANSM) was requested to find, identify and measure the active substances (ASs) contained in the product.

Materials and Methods At first, the analysis strategy was a general screening method to search for ASs in the product, performed with gas chromatography-mass spectrometry [GC-MS] and high performance liquid chromatography-mass spectrometry [HPLC$\mathrm{MS}$ ]. Then a specific method confirmed the identification and quantified the AS using ultra performance liquid chromatography-diode array detection [UPLC-DAD]

Results The slimming preparation was presented in capsules containing a fine, brown homogeneous powder. Gas Chromatography revealed two main ASs and the mass spectrometry analysis identified them as sibutramine and phenolphthalein. The result of HPLC/ MS also revealed two main ASs on chromatogram with molecular masses of 279 g.mol-1 and 318 g.mol-1. The UPLC-DAD, using the method 'search for and quantification of $34 \mathrm{ASs}$ in a slimming formulation', confirmed these preliminary results and also gave a quantity of $8 \mathrm{mg}$ of sibutramine and $20 \mathrm{mg}$ of phenolphthalein per capsule.

Conclusions Sibutramine is the AS in Sibutral (10 and $15 \mathrm{mg}$ ), an anti-obesity medicine, withdrawn from the market in January 2010 because of increased cardiovascular risk and an unfavourable benefit-risk assessment. Because of its carcinogenic potential phenolphthalein (a laxative) has been forbidden in France since 1999. Sibutramine and phenolphthalein were probably responsible for the clinical symptomatology in this patient. These slimming products sold outside the pharmaceutical distribution network have not been approved by the authorities resulting in a health risk, including fatal outcomes.

No conflict of interest.

\section{TCH-002 ANALYSIS OF DISPENSING LOGISTICS PROCESSES CARRIED OUT BY SEMIAUTOMATIC CAROUSEL SYSTEMS}

doi:10.1136/ejhpharm-2013-000276.193

M Martín-Herranz, MT Rabuñal-Alvarez, I Pedreira-Vázquez, S González-Piñeiro, M Calvin-Lamas, JR Vizoso-Hermida. Complejo Hospitalario Universitario A Coruña, Pharmacy, A Coruña, Spain

Background Hospital Pharmacy Services work hard at logistics to supply medicinal products to inpatients. This prompted the need to modernise the technical resources and logistical processes with a semiautomatic carousel system (SCS) for storing and dispensing.

Purpose To describe the logistics processes performed by our semiautomatic vertical and horizontal carousel systems (SVCS, SHCS) of the Kardex type.

Materials and Methods Descriptive observational study in a tertiary level hospital (1493 beds). Quantitative variable: 'medicines lines dispensing', defined according to the Product Catalogue and Invoicing Update from the SEFH-TECNO group (Spanish Society of Hospital Pharmacy-Evaluation Group of New Technologies). Study period: January-June 2012. Data Source: Pharmacy Service Internal Register computer application and Mercurio application version 2.12. The type of logistic process performed for dispensing is classified according to the type of order: stock in clinical unit, preparation of unidoses and replacement drugs for the Pyxis. The workload was calculated for each type of carousel according to the storage volume of each system $\left(\mathrm{SVCS}=15.6 \mathrm{~m}^{3}\right.$, SHCS $\left.=111.4 \mathrm{~m}^{3}\right)$.

Results The total number of lines dispensed during the study period was 1264751: 1235662 were prepared with SCS $(97.7 \%)$. Depending on the type of order, more work corresponded to the preparation of unidoses with 1128343 lines (91.31\%), followed by 83092 to prepare stock lines in clinical units (6.72\%) and 24227 order lines for stocking the Pyxis (1.96\%). Preparation of the unidoses was fully developed in the SVCS, while preparation of replacement stock for Pyxis and stock in clinical units were carried out in the SHCS. Depending on the type of carousel, the SVCS workload was 396.32 lines $/ \mathrm{m}^{3} /$ day, compared with 5.27 lines $/ \mathrm{m}^{3} /$ day for SHCS.

Conclusions Identifying and quantifying the processes undertaken by the SCS is a very useful tool that allows us to adjust the workloads of the pharmacy technicians.

No conflict of interest.

\section{TCH-003 ASSESSMENT OF AUTOMATED DRUG DISPENSING SYSTEM PERFORMANCE INDICATORS}

doi:10.1136/ejhpharm-2013-000276.194

I Blasco-Mascaro, G Mercadal-Orfila, R Romero-Del Barco. Hospital Mateu Orfila Pharmacy, Mahon, Spain

Background Hospital Mateu Orfila has approximately 140 beds. Since 2007 it has operated an automated drug dispensing system (Pyxis) comprising nine units, five of them linked to the electronic prescribing system (EP).

Purpose To assess performance indicators of the automated drug dispensing system (ADS) that can be used to monitor the effectiveness of processes within the hospital quality system. 